\title{
LA PRODUCCIÓN DE UN DISCURSO SEXUAL EN EL PROGRAMA DE ATENCIÓN INTEGRAL A LA ADOLESCENCIA
}

\section{THE PRODUCTION OF A SEXUAL DISCOURSE AT THE ADOLESCENCE INTEGRAL ATTENTION PROGRAM}

\author{
Isabel Gamboa Barboza*
}

\begin{abstract}
RESUMEN
Este trabajo hace un análisis de contenido de las dos técnicas propuestas por el PAIA (Programa de Atención Integral a la Adolescencia) de Costa Rica, para la atención en salud de las y los jóvenes, el test de tanner y el tamizaje.

El razonamiento principal que se propone es que el uso de estos instrumentos es un medio para favorecer un modelo sexual que inhibe ciertos deseos y actos por considerarlos poco saludables. Así, el PAIA, mediante el ejercicio de una política pública que se declara a favor de las y los adolescentes, termina ejerciendo dispositivos de control $y$ vigilancia que pretenden producir una "sexualidad saludable", con un fuerte contenido moral.
\end{abstract}

PALABRAS CLAVE: COSTA RICA * CUESTIONARIOS * ANÁLISIS CRÍTICO * PAIA * ADOLESCENTES * INTIMIDAD

\section{ABSTRACT}

This article analyzes the content of the two instruments proposed by Costa Rica's Adolescence Integral Attention Program (PAIA, for its Spanish acronym) to care for the health of adolescent women and men: the Tanner and the sifting tests.

The main hypothesis is that the use of these instruments is a means to favor a sexual model which inhibits certain desires and actions by considering them as non healthy. Therefore, the PAIA, through the exercise of a public policy which claims to favor adolescent women and men, applies control and surveillance mechanisms aimed at producing a "healthy sexuality", with a strong moral content.

KEY WORDS: COSTA RICA * QUESTIONNAIRES * CRITICAL ANALYSIS * PAIA * ADOLESCENT * PRIVACY

Programa Atención Integral en Salud, Universidad de Costa Rica.ilionee@hotmail.com 


\section{INTRODUCCIÓN}

Las personas jóvenes ${ }^{1}$ parecen ser las depositarias de un gran miedo cultural: se les presenta con una fuerte disposición al consumo de drogas, al sexo, a la vagabundería, a la inestabilidad emocional y a la autodestructividad ${ }^{2}$.

Estigmatizadas así, se justifican la construcción de mecanismos sociales, muchos de ellos, como los discutidos aquí, amparados en una política pública, para controlar esas características "indeseadas" y producir aquellas que se consideran aceptables; esto, según el discurso, con el propósito de proteger a las personas adolescentes de sí mismas.

Pero: ¿cuál es el mayor peligro del que se quiere proteger a las y los adolescentes? Quizá lo más inquietante, al nivel social, es la relación que se establece entre jóvenes, con las drogas, el sexo y la salud. De la correspondencia entre adolescencia, sexo y salud se ocupará este ensayo.

Aquellos aspectos relacionados con el sexo y la adolescencia parecen avivar, por decir lo menos, abundantes sentimientos de curiosidad y preocupación en la mayoría de las personas adultas.

El "sexo" y la "sexualidad" — construcciones culturales aún fuertemente vinculadas a la naturaleza - se representan como un todo (ejemplo de ello es la afirmación, frecuente en los discursos de profesionales en sexología, educación, medicina, en curas y en estudiantes, de que la sexualidad es todo: verse, tocarse, leer un libro, bailar ${ }^{3}$ ), como un todo que, como dice Joan Vendrell, generalmente interpelado desde el anacronismo, define nuestra identidad (Vendrell, 2004: 72-73).

Es por eso que cualquier deseo o práctica asociada a la "sexualidad" siempre resulta

1 A pesar de conocer las discusiones que suscita el uso de las palabras joven y adolescente, en este trabajo he decido, sin entrar en argumentaciones, usarlas como sinónimo.

2 La idea de las personas jóvenes como problema ha sido denunciada, en varias oportunidades, por el investigador Solum Donas; por ejemplo en Donas, Solum. "Salud integral y vida estudiantil". Adolescencia y Salud 1 (1). San José: EDNASSS. 1999: 29-34. un dato relevante. Ahora, si este deseo o esta actuación vienen de una persona definida como adolescente, el hecho cobra una importancia extraordinaria.

Los sistemas de salud no están exentos de esta obsesión con la "sexualidad adolescente", que es vista, en principio, como un problema o, dicho de manera muy directa, como algo patológico.

La población adolescente y su "sexualidad" son consideradas tácitamente como algo enfermo, lo que se comprueba en la creación de políticas, programas, instrumentos, entre otros, que procuran un acercamiento a las vivencias y pensamientos que dicha población tiene con respecto a la "sexualidad", con el fin de inculcar prácticas "saludables". Una de esas iniciativas es el Programa de Atención Integral a la Adolescencia (PAIA), creado en el año 1989, por la Caja Costarricense de Seguro Social (CCSS), con el fin de lograr que los servicios de salud que se ofrecen a las y los jóvenes tengan un carácter más apegado a sus necesidades ${ }^{4}$.

El PAIA, como parte fundamental de su quehacer, creó los dos cuestionarios analizados en este artículo: el Test de Tanner y las dos versiones del Tamizaje para adolescentes. El propósito de estas guías es conocer qué han pensado $y$ piensan, qué han sentido $y$ sienten, $y$ qué han hecho y harán, las personas jóvenes, con respecto a varios temas que más adelante se detallan.

La importancia de estos instrumentos radica en que se espera que los resultados obtenidos con sus aplicaciones, sirvan de base para determinar el estado de las y los jóvenes, sus necesidades en salud, $y$ para la formulación de políticas, programas y acciones dirigidas a este grupo.

El razonamiento que aquí se presenta incluye dos aspectos: el análisis del contenido

Para una discusión acerca de la importancia de la sexualidad en los discursos del Ministerio de Educación Pública (MEP) y la Iglesia Católica de Costa Rica, me permito referir a mi manuscrito: "El sexo como (lo)cura: el Hospital Psiquiátrico en Costa Rica. 1978-2004.” En dictamen.

4 Tomadode: $<$ http://www.binasss.sa.cr/adolescencia/ adolescenciacostarricense.htm\#AREAprioritaria> [consultado el 1/5/08] 
formal de dichos instrumentos, y, empíricamente, el análisis de algunos resultados de la aplicación de ambos test —con el único interés de ilustrar los instrumentos- en algunos Equipos Básicos de Atención Integral de Salud (EBAIS) del Área de Salud de Curridabat. Para ello, se hizo una selección, al azar de 32 expedientes, de un total de 1402 existentes a diciembre del año 2006, correspondientes a personas adolescentes atendidas en el Núcleo de Tirrases hasta la fecha ${ }^{5}$.

Este trabajo contiene, en primer lugar, la descripción general de los instrumentos de Tamizaje y el Test de Tanner $y$ de su contexto; seguidamente, una discusión sobre el carácter político, cultural e institucional de estos sondeos, incluyendo una breve información empírica sobre las personas que llenaron los instrumentos revisados; finalmente, el análisis de las preguntas relacionadas con la "sexualidad" y el sexo, contenidas en el Tamizaje y el Test de Tanner, a partir de los presupuestos teóricos de esta reflexión.

El artículo es resultado de una actividad propuesta en la planificación estratégica del Área de Salud de Curridabat, del Programa Atención Integral en Salud (PAIS), como uno de los aportes críticos del Área al PAIA.

\section{LOS INSTRUMENTOS}

En el año 2000, el PAIA termina la primera versión del Instrumento de Tamizaje para Adolescentes $^{6}$. Esta herramienta indaga sobre datos sociodemográficos, calidad de las relaciones familiares, comunicación, amistad, estados de ánimo, consumo de drogas, prácticas relacionadas con el sexo, entre otros aspectos.

Para el año 2006, el PAIA presenta una nueva versión del Tamizaje, agregando

5 Información ofrecida por la Unidad de Tecnologías de Información del PAIS. El Núcleo de Tirrases incluye los barrios La Colina, Lomas de San Pancracio, Ponderosa y Quince de Agosto.

Información ofrecida por el licenciado Carlos Garita Arce, funcionario del PAIA. Cita con su autorización. preguntas sobre "explotación sexual comercial" $y$ problemas alimenticios.

El otro cuestionario utilizado por la CCSS es una medida internacional conocida como Test de Tanner ${ }^{7}$. El Tanner hace preguntas sobre la percepción del propio cuerpo, conocimientos $y$ vivencias relacionadas con el sexo, entre otras cosas. Además, incluye representaciones gráficas donde se muestran los genitales masculinos o femeninos, para que la o el joven marquen aquellas figuras que más se asemejan a su cuerpo.

De ambos sondeos se recuperará lo relacionado directamente con los temas cercanos a la "sexualidad".

El PAIS ${ }^{8}$, en cumplimiento con la normativa, explícita en las fichas técnicas ${ }^{9}$, que define a las y los jóvenes como uno de los subprogramas meta, utiliza ambos instrumentos en la población entre 10 a 19 años de edad. Esto se hace preferiblemente durante la consulta clínica, pero también en centros educativos $y$, en algunas ocasiones, en las casas donde vive alguna o algún joven.

En general, cualquier persona del EBAIS puede encargarse de explicar y entregar las hojas a la población de jóvenes - que deben contestarlas de manera privada- pero es al personal médico a quien le incumbe realizar la interpretación de los resultados.

$7 \quad$ Fue imposible saber con exactitud la fecha en que este Test comenzó a usarse en el país. El licenciado Garita dice que esto fue desde antes de 1990. Agregó, que debió hacerse una validación o adecuación del Test para el país, pero esto no sucedió. Cita con su autorización.

8 Este programa es producto de un convenio firmado en el año 1999, entre la CCSS y la Universidad de Costa Rica, para que esta constituyera y pusiera en práctica un modelo de atención en salud para el primer nivel en los cantones de Curridabat, Montes de Oca y en los distritos de San Ramón, San Diego, Concepción y San Juan de la Unión.

$9 \quad$ La Ficha Técnica es un documento, elaborado por la Dirección de Compras de Servicios de Salud, de la CCSS, en el cual se especifican los indicadores que se incluyen en los Compromisos de Gestión que la CCSS negocia con las diferentes unidades de salud del país. 
Cuando el documento se entrega durante la consulta, la explicación y negociación para que sea llenado se hace directamente con la o el joven. Cuando es en otro espacio, se procura obtener el permiso de la madre o el padre ${ }^{10}$.

La idea es que los resultados que se obtengan con ambos sondeos sirvan para un "abordaje adecuado" de este grupo, por parte del personal de salud, que tiene la responsabilidad de educar en aquello que sea necesario para una adolescencia "saludable". El problema es que al carácter prescriptivo de lo "saludable" se añade la rigidez de la concepción de la adolescencia, que según el PAIA, es estar en:

[...] el período crucial del ciclo vital en que los individuos toman una nueva dirección en su desarrollo, alcanzan su madurez sexual, se apoyan en los recursos psicológicos y sociales que obtuvieron en su crecimiento previo, asumen para sí las funciones que les permiten elaborar su identidad $y$ plantearse un proyecto de vida propio. (Krauskopf, citada por CCSS, 2005: 80).

Es justo decir que quienes laboran o han trabajado en el PAIA han manifestado una gran preocupación por deconstruir la imagen tradicional de adolescencia; sin embargo, esto no se termina de lograr: la conceptualización que aceptan sitúa a la adolescencia más o menos igual que los discursos más tradicionales: como un momento, fase o etapa importantísima para la construcción de un proyecto hacia la adultez.

Esto no es extraño. El trabajo respetuoso con adolescentes es casi un imposible en la medida en que los proyectos de trabajo con esta población parten de un punto de vista conservador, es decir, buscan la transformación de las $y$ los adolescentes en "otros".

Reparemos ahora en los significados y las implicaciones de estos mecanismos de evaluación.

10 Estos procedimientos son los que, de manera general, se siguen en el Área de Salud de Curridabat. Información obtenida del señor Juan Carlos Morera Guido, médico Coordinador del Área de Salud de Curridabat. Cita con su autorización.

\section{"UNA SOCIEDAD SINGULARMENTE} CONFESANTE"

El Tamizaje y el Test de Tanner cumplen, aunque no únicamente, el papel de dispositivos de "confesión": con ellos, se espera estimular a las personas jóvenes para que escriban sobre todas sus fantasías, deseos, prácticas y temores.

Esto es lo que Michel Foucault denominó el efecto panóptico: la producción de mecanismos de observación cotidiana y detallada con los cuales se acaba produciendo diversos discursos sexuales (2002a:199-230). Se trata, según Foucault, de saber: "[...] quién es; dónde debe estar; por qué caracterizarlo; cómo reconocerlo; cómo ejercer sobre él, de manera individual, una vigilancia constante, etc. [...]" (2002a: 203).

Que seamos "una sociedad singularmente confesante" (Foucault, 2002b: 74) es posible debido a que, como lo señalaron Agnes Heller y Ferenc Fehér, el enfoque político actual de la salud pública considera la vida privada de las personas como un asunto de interés público (Heller y Fehér, 1995: 64) ${ }^{11}$. Este interés, a su vez, parte del supuesto de peligrosidad que conlleva toda acción privada. Probablemente, ya no podamos creer aquello de que "Dios perdona el pecado pero no el escándalo", o de que "pecar en secreto no es pecar".

Entonces, una pregunta que no podemos dejarnos de hacer - no formularla equivale a naturalizar lo que, por lo menos desde una perspectiva sociológica, debería ser interrogado- es: ¿qué hace posible que las personas adultas se sientan con el derecho y la capacidad de reunirse para elaborar instrumentos con los cuales preguntan a las personas jóvenes sobre su vida privada?

Probablemente, esos mecanismos son elaborados por personas que se atribuyen una superioridad moral e intelectual sobre personas estereotipadas como incapaces de custodiarse a sí mismas; gente que está legitimada por un

11 Para una discusión sobre el carácter público de la intimidad, léase: Berlant, Lauren y Michael Warner. "Sexo en Público". Rafael Mérida (ed). Sexualidades transgresoras. Una antología de estudios queer. Barcelona. Icaria, 2002: 229-257. 
supuesto saber y autocontrol del propio cuerpo; individuos que asumen, a lo mejor inconscientemente, que las y los jóvenes son personas "peligrosas" para sí mismas.

Pero el Tamizaje y el Test de Tanner también se corresponden con un contexto biopolítico donde la salud se ha vuelto una imposición moral (Heller y Fehér, 1995: 63-64), y los derechos colectivos —en este caso, de la población adolescente- suelen volverse una obligación (Heller y Fehér, 1995: 28). Sí, la salud —que durante la adolescencia es sobre todo "salud sexual y reproductiva" - ha pasado de ser un derecho a ser un deber que las y los jóvenes debe cumplir con la supervisión adulta.

La estrategia que permite este tipo de operaciones - mediante las cuales un grupo se reúne para analizar, clasificar y prescribir a otro-puede ser explicada a partir de lo que van Dijk ha revelado para los casos de racismo: se diferencia al grupo, se presenta esa diferencia como desviación de la norma, y se convierte la desviación en amenaza (Van Dijk y otros, 2000: 244).

Desde la introducción se señaló cómo las personas jóvenes son estereotipadas y estigmatizadas por las diferentes instituciones sociales $y$, por qué no, muchas veces a partir del sentido común de quienes allí trabajan.

Relacionado con esto, es necesario enfatizar en que el objetivo oficial o formal de estos documentos es el de detectar a las personas jóvenes que se encuentren en algún tipo de "riesgo para sí", con el fin de atenderles a tiempo con un enfoque preventivo:

[...] discriminar el riesgo psicosocial, de un posible daño específico de alta incidencia en los y las adolescentes. De tal forma que permita al personal de salud que lo administre, tomar acciones eficaces para evitar que el daño llegue a grados disfuncionales que vayan en detrimento de la población adolescente (Instrumento de Tamizaje para adolescentes. Instructivo smd).

Instituciones religiosas, educativas $y$ de salud, tienen como uno de sus propósitos fundamentales educar a la población adolescente en diferentes temas para que puedan vivir "responsablemente"; es lo que Deborah Britzman llama "discursos de información" (Britzman, 2002: 197-228), dirigidos frecuentemente a poblaciones consideradas vulnerables.

No se afirma aquí que quienes trabajan con adolescentes lo hagan por alguna clase de "mala fe"; las motivaciones individuales que una persona adulta tenga para laborar con esta población, no son de interés en este artículo; sí lo son las representaciones y los dispositivos de adolescencia, salud y sexualidad, que hacen posible que en este tiempo y esta cultura las personas adolescentes sean un tema.

El hecho de que se representa a las y los jóvenes como un problema de salud pública queda más claro si ubicamos ese discurso en un contexto más general —el de la construcción cultural de la sexualidad- del que se hablará en un momento. Y esto nos da para suponer que el riesgo que se quiere evitar, con la aplicación de estos sondeos, es el que representan estas personas para la sociedad. Veamos algunos resultados de los expedientes analizados.

La cantidad de adolescentes clasificados con alto riesgo, según los sectores ${ }^{12}$ del núcleo seleccionado, son: el sector dos con 25 expedientes, el sector 13 con 24 , el 14 con 13 atenciones, $y$ el sector uno con 36 expedientes, para un total de 98 adolescentes clasificados como de "alto riesgo". De estos, se estudió ocho, ocho, seis $y$ diez, respectivamente, para un conjunto de 32 expedientes clínicos examinados.

De los 32 , tal y como vemos en el gráfico 1, la cantidad de expedientes pertenecientes a adolescentes hombres que consultaron es inferior a los correspondientes a las adolescentes mujeres pues, un $56 \%$ concierne a éstas y un $44 \%$ a hombres ${ }^{13}$.

12 Las áreas de salud se dividen en Núcleos, que son la suma de dos o más EBAIS, cada EBAIS está ubicado en un sector, es decir, en una zona geográfica establecida por la CCSS.

13 En la población joven, al igual que en la adulta, la mayoría de personas que solicitan los servicios del EBAIS son mujeres, dicho de otro modo, los hombres en general presentan menos disposición para pedir la atención en dichos centros. Esto se debe, entre otros factores, a las expectativas que, cultural e individualmente, se tienen acerca 
El dato de que son mayoritariamente mujeres quienes acuden a los servicios de salud del EBAIS, más que una importancia demográfica en sí, es relevante pues significa que las mujeres jóvenes presentan una mayor "exposición" a los "discursos de información"; pues eso es lo que se espera: cuando el personal piensa que está frente a una o un adolescente con una señal de "riesgo", debe hablarle, explicarle por qué está en esa situación, y aconsejarle sobre cómo puede mejorar su situación.

\section{GRÁFICO 1}

\section{SEXO DE LAS PERSONAS ATENDIDAS}

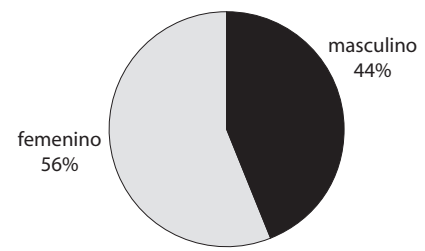

Fuente: Elaboración propia a partir de una muestra de los resultados del Test de Tanner aplicado a jóvenes del Núcleo de Tirrases durante el año 2006.

Como aparece en el gráfico 2, las edades de las personas cuyos expedientes se exploró, van desde los 10 años hasta los 20. En los extremos hay dos niños de 10 años, otro de 11 y una niña de 12 años; como se ve, la mayor concentración de edades es entre los 15 y los 20 años.

de cómo deberían ser y comportarse los hombres $y$ cómo las mujeres. En la medida en que estas son más estimuladas a mostrar comportamientos asociados a la debilidad o vulnerabilidad, pero además, en que sus condiciones de vida, materiales y psíquicas, son generalmente más hostiles, $y$ en que esto se traduce muchas veces en dolencias físicas, es de esperar que acudan más a los servicios sanitarios. En el caso de los hombres, se ha comprobado una relación entre masculinidad y riesgo para la salud: la demanda de fortaleza y aguante, aunque no puede aplicarse a todos los hombres pues las identidades, las prácticas sexuales y las estéticas, son muy diversas en la configuración de las masculinidades, aún puede implicar para muchos que visitar un servicio de salud equivalga a una feminización, o sea, una debilidad que cuestionaría su virilidad. Una investigación interesante sobre la relación entre masculinidad y cuerpo, que reflexiona sobre la necesidad constante en los hombres por demostrar su hombría, incluyendo la ausencia de afectos $y$ la pretendida invulnerabilidad, puede leerse en Rodríguez, María Elena. "Masculinidad y cuerpo: una paradoja”. Revista de Ciencias Sociales 76. San José. EUCR, 1997: 42-87.

\section{GRÁFICO 2}
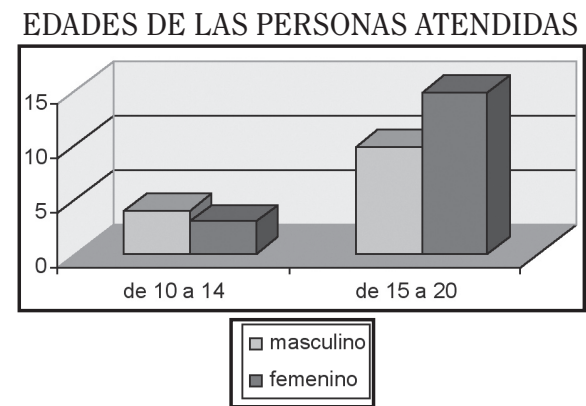

Fuente: Elaboración propia a partir de una muestra de los resultados del Test de Tanner aplicado a jóvenes del Núcleo de Tirrases durante el año 2006.

Esto no corresponde con que los límites de edades que entran en la definición del PAIA de adolescencia sean de los 10 años hasta los 24 (CCSS, 2005: 14), por alguna razón el Tamizaje incluye la aclaración de que debe ser llenado por adolescentes de 10 hasta 19 años.

\section{INTERROGANDO AL SEXO}

En el año 2002 , mediante el voto $\mathrm{N}^{\circ} 3759-02^{14}$, la Sala Constitucional declara con lugar un recurso de amparo interpuesto por la madre y el padre de dos mujeres menores de edad, ordenando a dos altos funcionarios de la CCSS eliminar del expediente médico de las menores el Test de Tanner que se les había aplicado, sin dejar copia del mismo; igualmente se condenó a la ccss al pago de los daños, prejuicios y las costas causados (Sala Constitucional de la Corte Suprema de Justicia, 2002). El recurso fue interpuesto, por esa madre y ese padre, debido a que, según apelaron, no se les pidió permiso para la aplicación del instrumento...

Lo anterior es mencionado como una muestra del forcejeo, por el control de la "sexualidad" de las personas adolescentes, por parte de diversas instituciones, en un intento por ubicar el tema en su contexto más allá de las instituciones de salud.

14 Agradezco a la licenciada Erika Linares Orozco y al licenciado Fernando Chavarría Lizano por facilitarme el material sobre esta resolución. 
Ese, es un hecho sugerente, dado que manifiesta una lucha de poder entre dos instituciones que se disputan la prerrogativa para definir quién debe administrar la "educación sexual" a la población joven, quién debe o puede hablarles del tema: la familia o el sistema de salud. Además, en la querella, entra a arbitrar un órgano judicial, que sentencia a favor de la primera, alegando que no se puede "burlar" "las potestades $y$ atribuciones que otorga la patria potestad de los progenitores" (Sala Constitucional de la Corte Suprema de Justicia, 2002). Esta relación de competencia entre la familia y los profesionales de salud, no debe hacernos olvidar que existe también una complicidad en el objetivo común de disciplinar a la juventud.

Pero: ¿cuál es la noción de sexo y sexualidad que se está produciendo en la elaboración y puesta en práctica de los test analizados? Para entender esto se retomarán algunos planteamientos de Gayle Rubin. Para ella, las sociedades occidentales producen y legitiman, de manera generalmente inconsciente, los siguientes axiomas acerca del sexo: el sexo es algo natural, sin ninguna relación con aspectos culturales; el sexo es potencialmente malo o peligroso; el sexo es algo muy importante que tiene consecuencias negativas; existe una jerarquía sexual según la cual lo mejor o bueno es el sexo llevado a cabo por amor, entre dos personas de distinto sexo, adultas, $y$ en una relación de pareja, $y$ lo peor o malo son todas aquellas prácticas sexuales entre personas del mismo sexo, desconocidos, o por dinero; finalmente, es necesario poner límites que distingan claramente entre la "buena" y la "mala" sexualidad, con el fin de evitar daños a las personas (Rubin, 1989: 130-143).

El contexto de las preguntas que vienen en el Tamizaje y el Test de Tanner se corresponde con los axiomas anteriores, es decir, no son cuestiones inofensivas o insignificantes, son interrogaciones que parten de la concepción de salud moralizante de la que hablan Heller y Fehér, que se adjudica la definición de pensamientos, sentimientos $y$ actos saludables -mejor dicho "buenos" $-y$ otros insalubres - o "malos" - en las experiencias sexuales de la gente, $y$ que ilustran la vigencia de esa "incitación para hablar del sexo" cuyo origen ubica Foucault en el siglo XVIII:
Se debe hablar del sexo, se debe hablar públicamente $y$ de un modo que no se atenga a la división de lo lícito y lo ilícito, incluso si el locutor mantiene para sí la distinción (para mostrarlo sirven esas solemnes declaraciones liminares); se debe hablar como de algo que no se tiene, simplemente, que condenar o tolerar, sino que dirigir, que insertar en sistemas de utilidad, regular para el mayor bien de todos, hacer funcionar según un óptimo. El sexo no es cosa que sólo se juzgue, es cosa que se administra. (Foucault, 2002b: 33-34).

La CCSS, $y$ en su interior el PAIA, no han podido, por así decirlo, salirse de los discursos hegemónicos sobre la "sexualidad" y la adolescencia. De manera que, aunque claramente hacen esfuerzos por tener un enfoque de trabajo más respetuoso y menos adultocéntrico, acaban reforzando aquello que critican.

En la primera versión del Tamizaje se indaga, en orden de aparición, sobre el número de personas con las que ha tenido relaciones sexuales en los últimos 6 meses; si durante la última relación sexual su pareja, o el-ella, usó un condón; $y$ si ha practicado alguna vez relaciones sexuales.

Para la segunda versión, que está apenas empezando a ser usada en algunos centros de salud del país, las preguntas, relacionadas con la sexualidad, que se incorporan, son: si ha tenido relaciones sexuales a cambio de dinero, comida, ropa, drogas; si ha sido obligada u obligado a realizar actos sexuales (aquí se detalla: "desnudarse ante otras personas, ver personas desnudas, ver películas pornográficas") o a tener relaciones sexuales; además, la cuestión de si ha tenido relaciones sexuales viene especificada: "tocamientos, penetración anal, penetración vaginal, sexo oral". La conclusión que salta a la vista inmediatamente es que el nuevo Tamizaje aumenta el número de temas sexuales $y$ desmenuza uno de los existentes; esto puede significar, entre otras cosas, que se aumentan las posibilidades de que una persona joven sea "problemática".

Mientras, en el Test de Tanner dirigido a las mujeres, se pregunta sobre la fecha de la 
primera menstruación, los días transcurridos entre esta y la segunda, la duración del sangrado, la fecha de la última regla, si presenta dolores u otro tipo de molestias cuando está con ella, si tiene alguna otra secreción vaginal, si considera la masturbación como algo normal, si ha practicado relaciones sexuales, si usa método anticonceptivo, si tiene problemas asociados al sexo que desee aclarar, y si ha sufrido abuso sexual.

En el caso del Tanner para los hombres se cuestiona sobre si ha notado cambios en el pene y testículos, si sabe qué es la emisión nocturna, si cree que la masturbación es algo normal, si ha practicado relaciones sexuales, si tiene dudas sobre el sexo que quiera hablar, y si ha sufrido abuso sexual.

Por último, y para poner un poco más en contexto al Test de Tanner, comentar que este tiene una nota, dirigida a las personas que lo llenarán, en la cual se afirma: "La sexualidad es una parte importante en la vida de toda persona. Sabemos que a veces es difícil hablar de ella, por lo que le solicitamos que nos conteste las siguientes preguntas"15.

La paradoja es indudable: por un lado, el reconocimiento de que la "sexualidad" es un tema difícil y privado, por el otro, la demanda para que se revele esa intimidad.

¿Por qué se indaga en un tema "privado" $y$ "difícil”? ¿De quiénes se quiere proteger esa intimidad? ¿Quiénes sí están autorizados a saber sobre la vida privada de estas personas? Por lo menos en el ámbito de la salud, todo el personal tiene la autoridad para preguntarle a alguien si usa condón, si le han penetrado analmente, si le parece que la masturbación es algo normal, etc.

Dicho lo anterior, reparemos en el énfasis reproductivo de estos test: uso de anticonceptivos, menstruación, vulva, pene, secreciones vaginales, emisiones nocturnas, tenencia $y$ frecuencia de relaciones sexuales, son los temas sobre los que más interesa indagar en ambos instrumentos.

El hecho de que, en el Test de Tanner, a las mujeres se les formula casi el doble de

15 Recuerde aquí que se supone que el Test es llenado en privado. preguntas que a los hombres, probablemente está relacionado con el papel fundamental que socialmente se ha otorgado a estas en la reproducción.

Igualmente, el que en ese instrumento se pregunte sobre el uso de anticonceptivos solamente a las mujeres, refuerza esa división sexual que les delega la responsabilidad de la reproducción.

Otra preocupación que se manifiesta mediante estos instrumentos es el saber qué tanta información posee la juventud. Esto puede estar relacionado con la idea de que entre más y "mejor" información tengan las personas jóvenes, más "protegidas" estarán. Y en ello, aunque usando diferentes mecanismos y contenidos, estarían de acuerdo diferentes instituciones tales como la iglesia católica, el Ministerio de Educación Pública y algunas organizaciones civiles: en que la "educación de la sexualidad" es una salida a los "problemas" relacionados con la sexualidad en los jóvenes de ambos sexos ${ }^{16}$.

Agregado a lo anterior, el preguntar por la cantidad de personas diferentes con las que se ha tenido sexo, es una de las manifestaciones de ese interés cultural y persistente en saber cuándo, cómo, cuánto, dónde, por qué, tiene sexo la gente joven ${ }^{17}$.

Finalmente, el Test de Tanner, al inquirirle a la persona entrevistada si tiene algo que quiera decir, lo hace de manera diferente para los hombres y para las mujeres. En el primer caso se pone: "¿Tiene alguna duda relacionada con el sexo que quiera hablar?", mientras a las mujeres se les pregunta: "¿Tiene algún problema relacionado con el sexo que quiere aclarar?"

Sobre el tema, refiero al manuscrito: "El sexo como (lo)cura..."

Esta fijación con el sexo y la juventud no es prerrogativa del sistema de salud, varias instituciones se pelean ese papel; entre ellas, los medios de comunicación, que con relativa frecuencia nos ofrecen "especiales" sobre el tema. Para ilustrar, cito el titular de un noticiero televisivo del 13 de marzo de este año 2007: "Los colegiales descubren el sexo en sus propias aulas". El título nos alerta sobre un peligro: el colegio no es el lugar seguro que los padres y las madres creen, hasta allí llega la amenaza del sexo. 
La diferencia es notoria en el sentido del lugar simbólico que otorga a unos y otras: para los hombres el lugar de la duda y del hablar, para las mujeres el lugar del problema y la aclaración.

Ahora bien, un aspecto fundamental del instructivo para el uso del Tamizaje, es el criterio de que si una persona adolescente ha tenido relaciones sexuales esto basta para clasificarla en riesgo. Incluso, hay expedientes donde esa era la única razón existente, no sólo para ubicar a esta persona como de riesgo sino incluso de "alto riesgo". Es por ello que el gráfico 3 revela que la mayoría de jóvenes incluidos aquí, 22 , han tenido al menos una relación sexual, ¿por qué creemos que sexo-adolescente $=$ problemas ${ }^{38} \mathrm{Es}$ importante aclarar que esta patologización del sexo en adolescentes comprende todo el período definido como adolescencia, de manera que no se trata, como podría pensarse, de casos de violación o abuso sexual. De eso se hablaba anteriormente, de que las personas adultas solemos tener actitudes muy conservadoras frente a las distintas experiencias sexuales.

\section{GRÁFICO 3}

\section{¿HA TENIDO RELACIONES SEXUALES?}

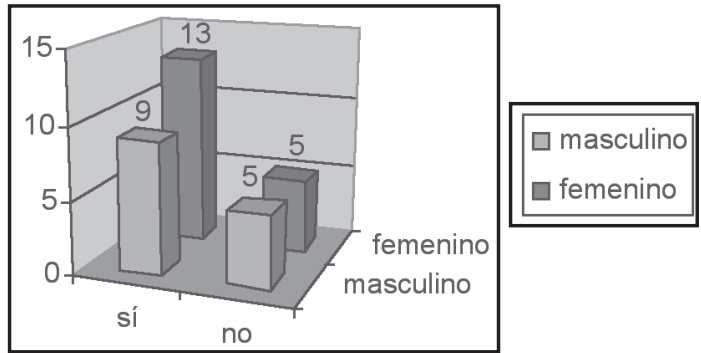

Fuente: Elaboración propia a partir de una muestra de los resultados del Test de Tanner aplicado a jóvenes del Núcleo de Tirrases durante el año 2006.

18 Un ejemplo de cómo eso no puede darse por sentado, lo encontramos en una investigación que realizó Joan Vendrell, con mujeres y hombres jóvenes, habitantes de Cuernavaca, México, en la que razona que estas personas: "No solo viven su 'sexualidad' de una manera personal, sino además de una manera 'despreocupada', 'desproblematizada'. Ninguno de nuestros interlocutores parece haber sentido jamás la necesidad de buscar información o apoyo de una forma más sistemática [...]" (Vendrell, 2005: 107).
El poder de la adultez legitima para producir una normatividad sexual, desde la cual se decide que la existencia de prácticas sexuales en adolescentes es un factor que les pone en riesgo. Detrás de esta idea se encuentra el pensamiento, del que nos advirtió Rubin, de que el sexo es algo malo que puede generar consecuencias negativas.

Es precisamente esa "negatividad sexual" la que nos ayuda a entender el que la cCss parta de un enfoque de "salud sexual y reproductiva" ${ }^{19}$; es decir, de una perspectiva que asume al sexo como algo que tiene consecuencias negativas por lo que es menester higienizarlo, volverlo "saludable", $y$ demarcarlo de lo no "saludable".

El afán regulador de la "salud sexual y reproductiva”, llega a manifestaciones paródicas cuando se establece, de forma tácita o explícita, con quién se puede tener sexo, en cuáles lugares, en qué condiciones, de qué manera, con cuánta frecuencia.

Así como se parte de que el sexo adolescente no es "saludable", $y$ por lo mismo se otorga gran importancia al dato de si quien llega a la consulta del EBAIS ha tenido, o no, relaciones sexuales, también se parte, implícitamente, de que la homosexualidad, la bisexualidad, el lesbianismo, etc. son insanos. Los sabemos no porque se diga abiertamente, sino porque se actúa, al dar por sentada la heterosexualidad de la población adolescente a entrevistar. Sí, estos dos instrumentos son, en palabras de Berlant y Warner, heteronormativos:

La heteronormatividad es más que una ideología, prejuicio o fobia contra los gays y las lesbianas; se produce en casi cada aspecto de las formas y los procedimientos de la vida social: nacionalidad,

19 La perspectiva del PAIA sobre lo que es la sexualidad (y por tanto la "salud sexual y reproductiva") coincide con la señalada por Vendrell (un todo central y que siempre ha existido): "La sexualidad en tanto dimensión inherente e inmanente del ser humano, se constituye en una área prioritaria de trabajo en la que se comprenden los diversos componentes de esta dimensión de la vida". (CCSS, 2005:156). 
Estado y ley; comercio; medicina y educación; se produce también en las convenciones $y$ afectos de la narratividad, el romance $y$ otros espacios protegidos de la cultura. (Berlant y Warner, 2002: 238).

Lo que se ha venido afirmando a lo largo de este trabajo puede constatarse de manera directa en las producciones discursivas del PAIA $^{20}$. Note usted la definición de "sexualidad sana" a la que se suscriben:

1. La capacidad para disfrutar de actividades sexuales $y$ reproductivas que se encuentren reguladas a partir de una ética personal y social, 2. la ausencia de factores psicológicos como elementos afectivos (temor, vergüenza, culpabilidad) o cognitivos (creencias infundadas, mitos, prejuicios) que inhiban la reacción sexual o perturben las relaciones sexuales (genitales y no genitales) y 3 . la ausencia de trastornos orgánicos, de enfermedades $y$ deficiencias que entorpezcan u obstaculicen la actividad sexual y reproductiva. (Cerruti y Behar, citados por Fallas y Valverde, 2000: 19).

Este discurso, inconfundiblemente sexológico, parte de que el sexo, visto además como un proceso que debe generar una reacción, debe tener: una dosis de voluntariedad; una finalidad, en este caso, el disfrute (como obligación), que implica gozar la relación sexual y no tener miedos, vergüenza u otro sentimiento que pueda obstaculizar ese deleite; una relación estrecha con lo reproductivo; $y$ un control o regulación para que sea "saludable".

Así, el PAIA da por supuesto algo que debería problematizar: la intromisión pública en la vida privada de una población, y la construcción

A pesar de la leyenda que viene en la contratapa del libro, en la cual se aclara que no son responsabilidad del PAIA las opiniones expresadas por los autores, sí son sus discursos, porque el PAIA no es un ente, es una institución conformada por las personas que ahí trabajan y deciden, o no, publicar ciertas ideas. de una determinada "sexualidad" como "saludable", obviando, como dice Foucault, que eso no responde a una demanda de las personas interpeladas:

En primer lugar, la medicina responde a otro motivo que no es la demanda del enfermo, cosa que sólo acontece en casos más bien limitados. Con mucha más frecuencia la medicina se impone al individuo, enfermo o no, como acto de autoridad. (Foucault, 1996: 75).

Sería ingenuo creer que, en verdad, el PAIA busca el beneficio de las personas jóvenes -lo que no quiere decir que pretenda perjudicarles-. El asunto de fondo es la fabricación de discursos y acciones para garantizar "estilos de vida saludables" que, a su vez, se traducen en una sexualidad aséptica, impuesta como una verdad indisputable.

\section{CONCLUSIONES}

El Test de Tanner y el Tamizaje Adolescente son instrumentos de análisis que parten de representaciones que reproducen nociones conservadoras y patologizadoras sobre la adolescencia, la sexualidad $y$ la salud. Estas ideas convierten en temas no solo a los deseos y las experiencias de las personas jóvenes, sino a dicha población en sî misma, que de esta forma es transformada en un tema-problema por "abordar" médicamente.

De tal manera, estos sondeos acaban produciendo, desde un punto de vista médicomoral, deseos, sentimientos, pensamientos y acciones que se catalogan como saludables para esta población en particular.

Son, por tanto, mecanismos que lejos de problematizar esta realidad, por ejemplo analizando los miedos, prejuicios y estereotipos que se cuelan en las vivencias de las y los jóvenes, los simplifican al universalizarlos, olvidando, como advierte Weeks, que el sexo no es ni bueno ni malo (1998: 83). Que lo bueno y lo malo lo define cada cultura, cada acción, cada abordaje disciplinar, en el entendido de que el límite siempre está dado por los derechos de toda persona a no ser sometida sexualmente. 
La fascinación que nuestra cultura contemporánea deposita en la población adolescente tiene un alto grado de hostilidad, notoria en las leyes, decretos, vídeos, manuales, programas radiales y televisivos, afiches, desplegables, calcomanías, $y$ un sinfín de grupos integrados por esta población, que son producidos por instancias estatales, religiosas, internacionales $y$ civiles, que tienen como fin decirle a esta gente como debe vivir.

El que estos instrumentos hacen las veces de dispositivos de control y producción moral, puede constatarse por el paradigma sexual que les fundamenta: uno que interpreta al sexo como un todo ahistórico y natural; excesivamente importante $y$ peligroso, sobre todo para la gente adolescente; $y$ que, por lo mismo, justifica una, también excesiva, intervención en la vida privada de las personas jóvenes.

En vez de esto, el PAIA podría facilitar espacios de discusión sobre las representaciones que el personal de salud tiene acerca de la sexualidad. También, promover una evaluación sobre los aportes que, en la práctica, dichos instrumentos ofrecen, o no. Además, podría liderar discusiones críticas sobre las categorías de análisis de las que el sistema de salud parte en su trabajo con adolescentes. En este diálogo, tendrían que estar, por supuesto, aquellas de las que se habla: las personas que aquí y ahora construimos como adolescentes.

\section{BIBLIOGRAFÍA}

Berlant, Lauren y Michael Warner. "Sexo en público”. Rafael Mérida (ed.). Sexualidades transgresoras. Una antología de estudios queer. 1era. edición. Barcelona. Icaria, 2002: 229-257.

Britzman, Deborah. "La Pedagogía transgresora y sus extrañas técnicas”. Rafael Mérida (ed). Sexualidades transgresoras. Una antología de estudios queer. lera edición. Barcelona. Icaria, 2002: 197-228.

Caja Costarricense de Seguro Social. Instrumento de Tamizaje para Adolescentes. (s.d.t.).
Test de Tanner. (s.d.t.).

. Bases Programáticas. Programa

de Atención Integral a la Adolescencia.

Segunda edición. San José: PAIA, 2005.

Donas, Solum. "Salud integral y vida estudiantil". Adolescencia y Salud 1 (1). San José. EDNASSS, 1999: 29-34.

Fallas, Hannia y Oscar Valverde. Sexualidad y salud sexual y reproductiva en la adolescencia. Módulo de capacitación para personal de salud. Guía metodológica. San José: PAIA, 2000.

Foucault, Michel. (1976). Historia de la sexualidad. La voluntad de saber. $2 \mathrm{da}$ edición. México: Siglo Veintiuno, 2002b.

La vida de los hombres infames. La Plata: Editorial Altamira, 1996.

. Vigilar y castigar. Nacimiento de la prisión. 1era edición. Argentina: siglo XXI, 2002a.

Gamboa, Isabel. "El sexo como (lo)cura. El Hospital Psiquiátrico en Costa Rica. 1978-2004". Manuscrito en evaluación.

Heller, Ágnes, Ferenc Fehér. La modernidad y la liberación del cuerpo. 1era edición. Barcelona: Península, 1995.

Instrumento de tamizaje para adolescentes. Instructivo. Formulario nro. 4-70-030550. Manuscrito sin más información.

Rodríguez, María Elena. "Masculinidad y cuerpo: una paradoja". Revista de Ciencias Sociales 76. San José. EucR, 1997: 42-87.

Rubin, Gayle. 1era edición 1989. "Reflexionando sobre el sexo: notas para una teoría radical de la sexualidad”. Carole S. Vance 
(comp.) Placer y peligro. Explorando la sexualidad femenina. Segunda edición. Madrid. Talasa, 1989: 130-143.

Sala Constitucional de la Corte Suprema de Justicia. Expediente 02-001194-0007-CO. 2002.

Van Dijk, Teun, Stella Ting-Toomey, Geneva Smitherman y Dense Troutman. "Discurso, filiación étnica, cultura y racismo". Teun van Dijk (comp.) El Discurso como interacción social. Estudios del discurso: introducción multidisciplinaria 2. 1era edición. España. Gedisa, 2000: 213-262.
Vendrell, Joan. "La centralidad de la sexualidad en la era moderna". Gloria Careaga y Salvador Cruz (eds.) Sexualidades diversas. Aproximaciones para su análisis. lera edición. México. PUEG, 2004: 72-73.

. "Sexualmente no identificados: aproximación al nomadismo sexual entre jóvenes mexicanos". Edith Peña y Joan Vendrell (eds.) Revista de Estudios de Antropología Sexual 1 (1), enero-diciembre. Morelos. Instituto Nacional de Antropología e Historia/Universidad Autónoma del Estado de Morelos, 2005: 93-111.

Weeks, Jeffrey. Sexualidad. México: Paidós, 1998. 